\title{
Analysis and Estimation of Excitation Conduction With Wolff-Parkinson-White Syndrome Patients Based on a 3-D Magnetocardiogram
}

\author{
K. Kobayashi, Y. Uchikawa, Member, IEEE, K. Nakai, and M. Yoshizawa
}

\begin{abstract}
The purpose of this paper is to evaluate the accessory pathway of the Kent bundle in the Wolff-Parkinson-White (WPW) syndrome. We measured three-dimensional (3-D) vector measurements of magnetocardiograms (MCGs) for normal subjects and patients with the WPW syndrome, using 3-D second-order gradiometers connected to 39 dc SQUIDs, which can simultaneously detect magnetic field components perpendicular to the chest wall (Bz) and tangential to the chest wall (Bx, By), respectively. Source location for the accessory pathway was estimated by 3-D magnetic field components using the equivalent current dipole method. The magnetic field map of the delta wave obtained from $\mathrm{Bz}$ was not helpful in estimating the location and the number of sources, owing to the lack of a dipole pattern. On the other hand, by referring the magnetic field maps of the delta wave obtained from $\mathrm{Bx}$ and $\mathrm{By}$, the multiple sources (two sources) overlapping in time were clearly estimated. We demonstrated that 3-D MCG measurements are effective for analyzing multiple sources overlapping in time.
\end{abstract}

Index Terms-Accessory pathway, source estimation, threedimensional magnetocardiogram, Wolff-Parkinson-White syndrome.

\section{INTRODUCTION}

$\mathbf{M}$ AGNETOCARDIOGRAM (MCG) measurement of the magnetic field perpendicular to the body surface is widely used. We had developed a SQUID system for threedimensional (3-D) magnetic field measurements [1]. In the Wolff-Parkinson-White (WPW) syndrome, the existence of the accessory pathway often caused paroxysmal atrial tachycardia. A recent therapeutic advance in cardiology, an interventional procedure using a catheter, has been adopted for an ablation of accessory pathways [2]. In this case, preoperative evaluation of the accessory pathway is needed for shortening of treatment and reducing the burden on the patient.

There are some MCG studies of the WPW syndrome in source estimation [3] and modeling of the WPW syndrome [4]. In this study, we performed a 3-D measurement of an MCG for normal subjects and for patients with the WPW syndrome.

Manuscript received February 14, 2002. This work was supported by the Joint Research Project for Regional Intensive in Iwate Prefecture, by the Toyota Physical and Chemical Research Institute, and under a Grant-in-Aid from the High Technology Research Center, Tokyo Denki University.

K. Kobayashi and M. Yoshizawa are with the Faculty of Engineering, Iwate University, Morioka, Iwate, Japan (e-mail: kobaya@iwate-u.ac.jp; yosizawa@ iwate-u.ac.jp).

Y. Uchikawa is with the Faculty of Science and Engineering, Tokyo Denki University, Hikigun, Saitama, Japan (e-mail: uchikawa@f.dendai.ac.jp).

K. Nakai is with the Laboratory of Medicine, Iwate Medical University, Iwate, Japan (e-mail: knakai@iwate-med.ac.jp).

Digital Object Identifier 10.1109/TMAG.2002.802283

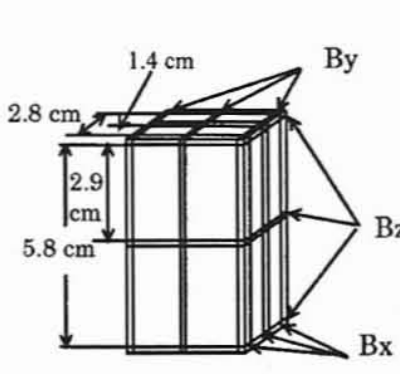

(a)

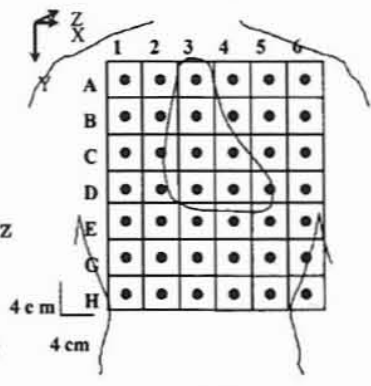

(b)
Fig. 1. Coordinate system and a 3-D second-order gradiometer and measurement of 48 positions on body surface. (a) The 3-D second-order gradiometer. (b) The 48 measurement positions.

In order to estimate the source of the accessory pathway of the WPW syndrome, the equivalent current dipole method was applied at the delta-wave period.

\section{METHOD}

Fig. 1 shows the configuration of a 3-D second-order gradiometer and the 48 measurement positions [fixed circle in Fig. 1(b)] on the body surface. The MCG of normal subjects and two patients with the WPW syndrome were measured by this system. Standard 12-lead electrocardiogram (ECG) was also measured. A band-pass filter was used in the range of $0.1-80 \mathrm{~Hz}$. All magnetic data were averaged for 150 times at each position. The reference signal for averaging was the $\mathrm{R}$-wave of the ECG (usually in lead II). The moving equivalent current dipole method was adopted for estimating the source location [5].

\section{RESULTS}

Fig. 2 shows an example of the MCG waveforms recorded from a patient with the WPW syndrome. In these waveforms, the delta wave that is a feature of the WPW syndrome exists. Fig. 3 shows the isofield contour maps that the delta wave exists (the vertical line in Fig. 2). Solid lines show the positive field in Fig. 1(b). For example, solid lines of the $\mathrm{Bz}$ component show magnetic flux that comes into the body.

As shown in Fig. 3(a), the isofield contour map of Bz shows one extreme at position $\mathrm{C} 4$. This isofield contour map, which did not indicate a dipole pattern, was not helpful in estimating the source location and the number of sources. On the other hand, in the contour maps of Bx and By shown in Fig. 3(b) and (c), 


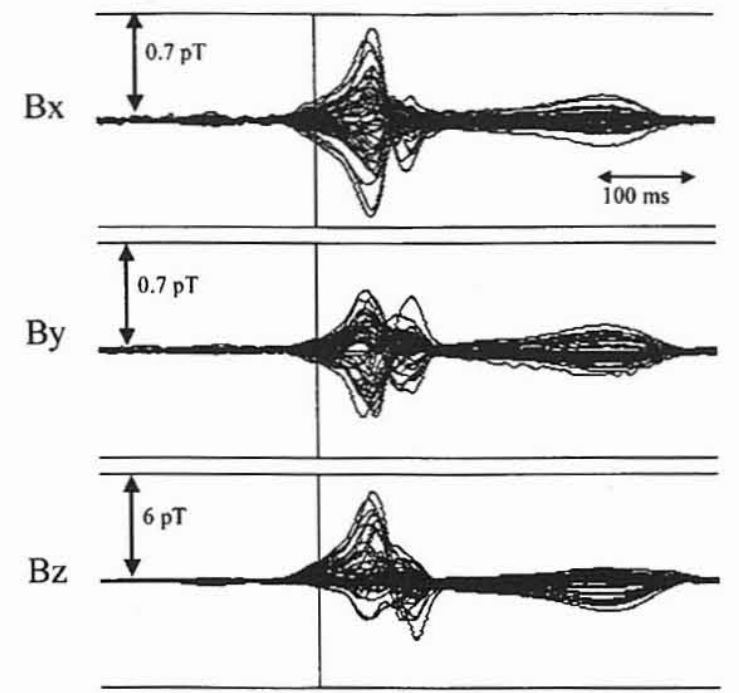

Fig. 2. MCG waveforms in a patient with the WPW syndrome.

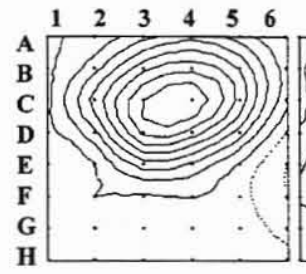

(a)

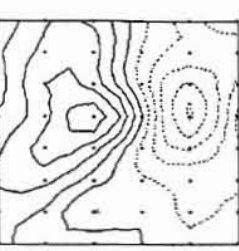

(b)

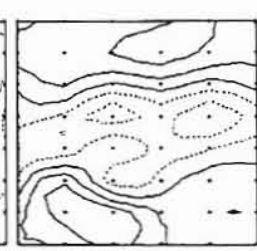

(c)
Fig. 3. Isofield contour maps of delta wave in the patient with the WPW syndrome. (a) Bz (130 fT/step). (b) Bx (25 fT/step). (c) By (25 fT/step).

respectively, we can clearly see two extremes at positions D3 and D5 on both contour maps. The characteristic of the tangential magnetic field pattern shows that a source exists under extreme value [5]. Namely, these magnetic fields consist of two sources underlying each extreme. We carried out source estimation using this information. Fig. 4 shows the chest X-ray superimposed source localizations. The arrow shows position and direction of estimated source. Two sources were estimated at right atrium lower (D3) and at left side between left atrium and left ventricle (D5).

\section{Discussions}

The ECG of the patient with the WPW syndrome shows road $\mathrm{I} \pm$, roads II and III +, roads aVR and $\mathrm{aVL}-$, road $\mathrm{aVF}+$, and roads $V_{1}-V_{6}-$. It is considered that the accessory path exists almost in the left side between the left atrium and left ventricle [6]. Therefore, it is proven that the estimated source in the left side between left atrium and left ventricle (D5) of Fig. 4 is the accessory path.

On the other hand, the source at right atrium lower (D3) of Fig. 4 seems to be catching the repolarization process of the right atrium. The MCG of normal subjects at the same time was analyzed for inspection of this result.

Fig. 5 shows an example of the MCG waveforms recorded from the normal subject. Fig. 6 shows the isofield contour maps

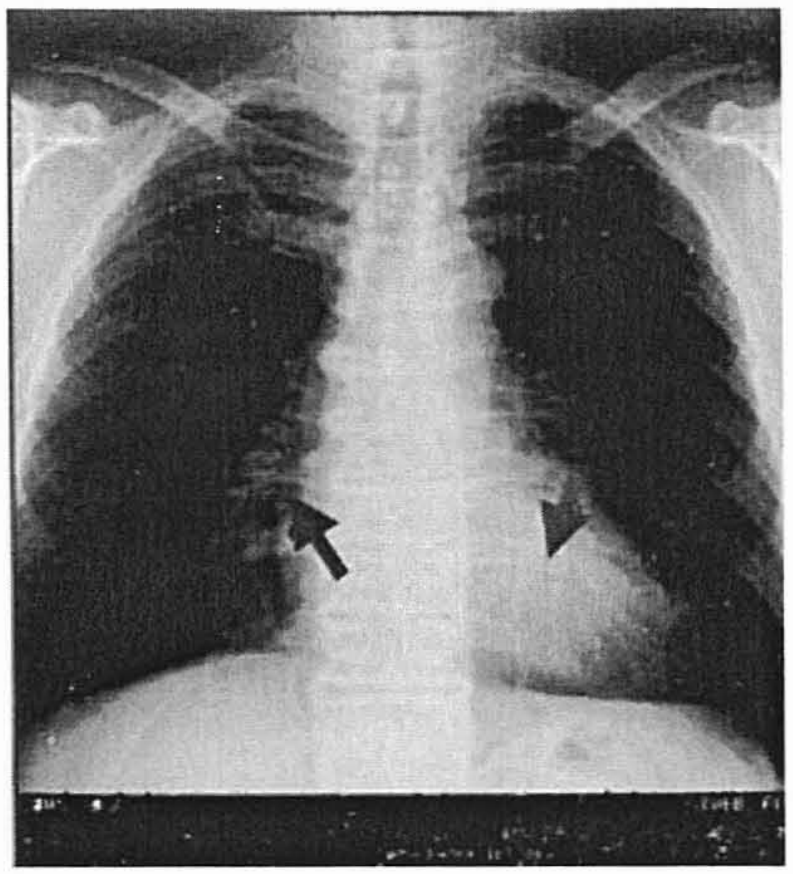

Fig. 4. Chest X-ray superimposed source localizations in the patient with the WPW syndrome.

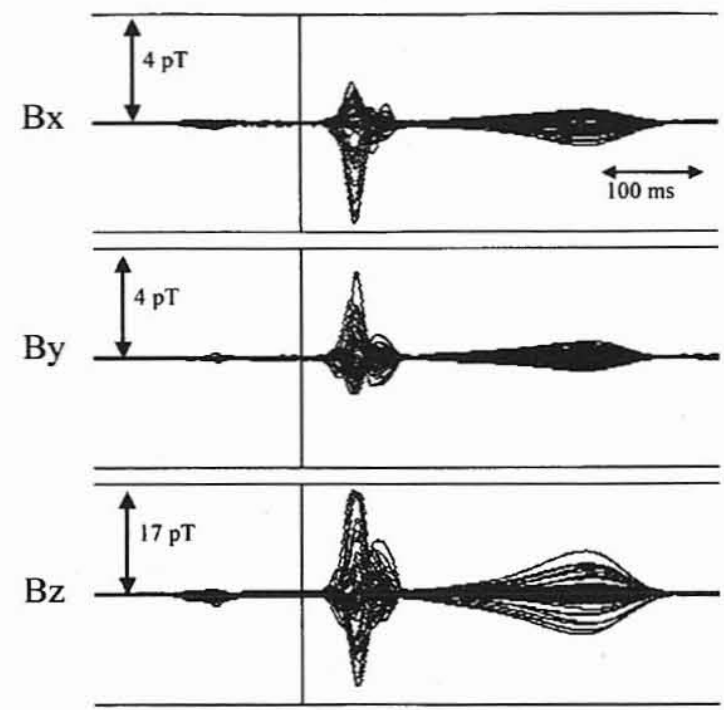

Fig. 5. MCG waveforms in a normal subject.

at the vertical line in Fig. 5. As shown in Fig. 6(a), the isofield contour map of Bz shows a dipole pattern. Fig. 7 shows the chest $\mathrm{X}$-ray superimposed single source localization calculated from Fig. 6. The source was estimated at the right atrium lower. In the normal subject, the activity of the heart in this time is the time in which the stimulation transmits to the His bundle, and even in the time of the repolarization of the atrium which is the early excitation site. Therefore, the source at right atrium lower (D3) in the WPW syndrome patient is proven with that repolarization process of right atrium. The estimation of the accessory pathway received the effect of repolarization process of right atrium. 


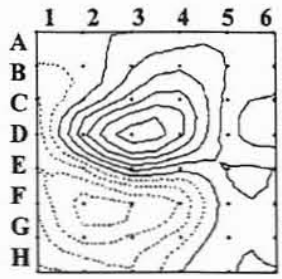

(a)

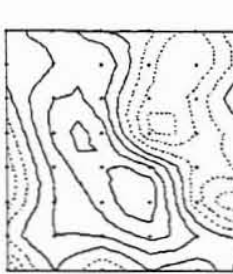

(b)

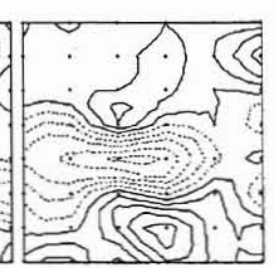

(c)
Fig. 6. Isofield contour maps in the normal subject. (a) $\mathrm{Bz}$ ( $77 \mathrm{fT} / \mathrm{step}$ ). (b) $\mathrm{Bx}$ (13 fT/step). (c) By (13 fT/step).

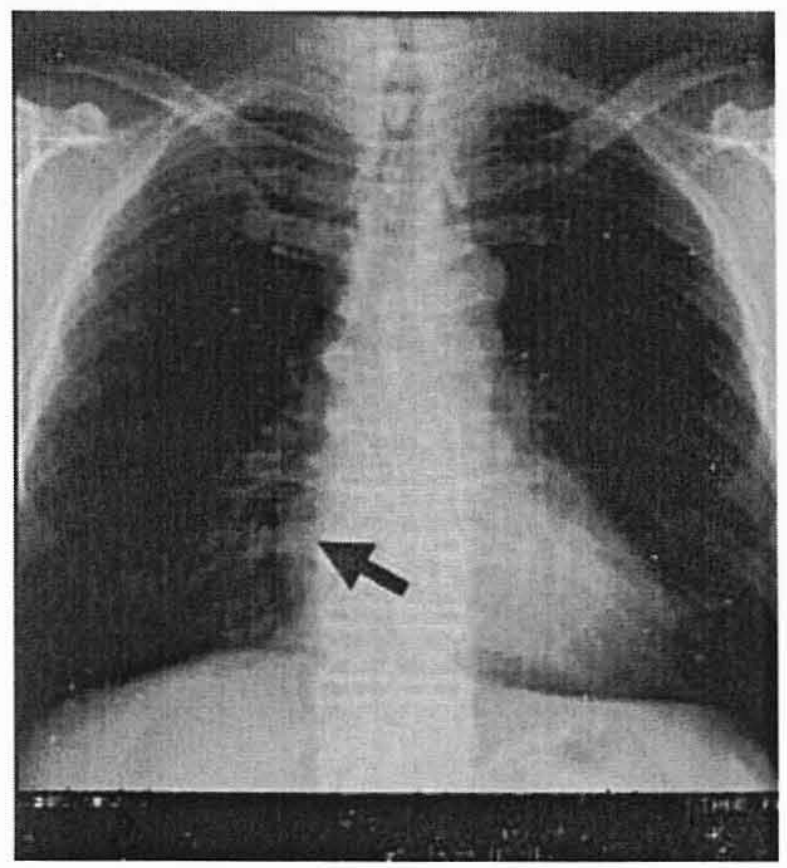

Fig. 7. Chest X-ray superimposed source localization in the normal subject.

Next, the estimation of this WPW patient in the same time (the vertical line in Fig. 2) was carried out by the assumption of single source. Table I shows source locations estimated by singlesource and two-sources methods. The result of the two-sources method indicates only the accessory pathway. The distance of these estimated sources is calculated. The distance of these es-
TABLE I

SOURCE LoCATIONS Estimated By Single-SOURCE AND TWO-SOURCES METHOD AND DISTANCE OF THESE SOURCES

\begin{tabular}{|c|c|c|c|c|c|c|c|}
\hline & \multicolumn{3}{|c|}{ single source } & \multicolumn{3}{|c|}{ two sources (accossory path) } & \multirow{2}{*}{$\begin{array}{l}\text { distance } \\
\text { Imm }\end{array}$} \\
\hline timoims & $\mathrm{X} / \mathrm{mm}$ & Y $1 \mathrm{~mm}$ & $\mathrm{Z} \mathrm{mm}$ & $x[\mathrm{~mm}$ & $\mathrm{Y} / \mathrm{mm}$ & $\mathrm{z} \mathrm{mm}$ & \\
\hline 450 & 66 & -18 & -79 & 58 & -19 & -76 & 9 \\
\hline 460 & 63 & -19 & -75 & 57 & -20 & -70 & 8 \\
\hline 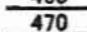 & 63 & -14 & -74 & 59 & -16 & -70 & 6 \\
\hline 480 & 64 & -10 & -73 & 61 & -12 & -67 & 7 \\
\hline 490 & 62 & -7 & -69 & 59 & -8 & -64 & 6 \\
\hline 500 & 66 & -4 & -65 & 64 & $\overline{-6}$ & -58 & 8 \\
\hline
\end{tabular}

timated sources is from 6 to $9 \mathrm{~mm}$. The goodness-of-fit of the single-source model is from $63 \%$ to $73 \%$; the goodness-of-fit of the two-sources model is from $75 \%$ to $82 \%$.

The utilization of the tangential magnetic fields in the 3-D measurement can provide detailed information of the source from measured data. The 3-D measurement is effective for the analysis of the activity of multiple sources.

\section{CONCLUSION}

We performed a 3-D MCG measurement in normal subjects and patients with the WPW syndrome. A 3-D measurement can estimate the accessory pathway and the repolarization process of right atrium. These findings demonstrated that the 3-D MCG measurements were valuable for the analysis of the heart activity.

\section{REFERENCES}

[1] K. Kobayashi and Y. Uchikawa, "Development of a three-dimensional biomagnetic measurement system with 39-channel," in Recent Advances in Biomagnetism. Tohoku, Japan: Tohoku Univ. Press, 1999, pp. $35-38$.

[2] K. Grauer, A Practical Guide to ECG Interpretation. Tokyo, Japan: Medical View, 1995.

[3] J. Nenonen, T. Katila, M. Leinio, J. Montone, M. Makijarvi, and P. Siltanen, "Magnetocardiographic functional localization using current multipole models," IEEE Trans. Biomed. Eng., vol. 38, pp. 648-657, July 1991.

[4] Y. Uchikawa and S. N. Erne, "Modeling the Wolfe-Parkinson-White syndrome for magnetocardiography," in Proc. Biomagnetism '87, pp. 322-325.

[5] K. Kobayashi and Y. Uchikawa, "Estimation of multiple sources using a three-dimensional vector measurement of a magnetoencephalogram," J. Appl. Phys., vol. 83, pp. 6462-6464, 1998.

[6] J. J. Gallagher, W. M. Smith, and J. H. Kassel et al., "Role of mahaim fibers in cardiac arrhythmias in man," Circulation, vol. 64, pp. 176-176, 1981. 